\title{
Twistors and induced representations of $\mathrm{SU}(2,2)$ *
}

\author{
David E. Lerner \\ University of Kansas, Lawrence, Kansas 66045 \\ (Received 18 October 1976)

\begin{abstract}
We give an explicit realization of a series of representations of $\operatorname{SU}(2,2)$ induced by $\mathbf{R}^{+} \otimes \operatorname{SL}(2, \mathbf{C})$. Vectors in these representation spaces are homogeneous spinor-valued functions of two twistor variables. They may also be realized, in a frame-dependent way, either as conformally invariant fields in Minkowski space or as homogeneous spinor-valued functions on the $O(2,4)$ null cone. The conformal invariance of the massless free fields is discussed from this point of view, and the twistor version of the field equations is derived. Finally, irreducible twistors are shown to correspond to conformally invariant fields satisfying the generalized twistor equation.
\end{abstract}

\section{INTRODUCTION}

Among the most important representations of the conformal group of space-time are those which give rise to physical fields in Minkowski space. These fields can be constructed by the method of induced representations; they are derived by means of a certain projection operation from the cross sections of homogeneous $\mathrm{SU}(2,2)$ vector bundles. In suitably chosen bases, these cross sections assume the familiar form of homogeneous spinor-valued functions on the $O(2,4)$ null cone; the actual physical fields are obtained by projecting (or restricting) these functions to Minkowski space. ${ }^{1-4}$

An alternative realization of some of these fields has been obtained by Penrose, ${ }^{5-7}$ who shows that to each set $\left\{\varphi_{r}: 0 \leqslant r \leqslant n\right\}$ of holomorphic functions of two twistor variables $U$ and $V$ satisfying

$$
U^{\alpha} \frac{\partial \varphi_{r}}{\partial U^{\alpha}}=-(r+1) \varphi_{r}, \quad V^{\alpha} \frac{\partial \varphi_{r}}{\partial V^{\alpha}}=-(n-r+1) \varphi_{r},
$$

and

$$
\frac{\partial \varphi_{r}}{\partial U^{\alpha}}+\frac{\partial \varphi_{r+1}}{\partial V^{\alpha}}=0
$$

there corresponds a unique real analytic solution to the zero rest-mass free field equations ${ }^{8}$

$$
\nabla^{A A^{\prime}} \varphi_{A^{\prime} B^{\prime} \cdots C^{\prime}}=0
$$

in Minkowski space. Conversely, it can be shown (Sec. 5 , see also Ref. 9 for an altogether different approach) that any real analytic solution to (1.3) may be described by holomorphic twistor functions.

More generally, it turns out that any representation of of $\mathrm{SU}(2,2)$ induced by $\mathrm{R}^{+} \otimes \mathrm{SL}(2, \mathrm{C})$ may be realized on a space of vector-valued functions of two twistor variables (Secs. 3 and 4). If the representation can be analytically continued to the complex $\mathrm{O}(2,4)$ null cone, then the real analytic fields determined by the representation will correspond to holomorphic twistor functions. It is a decided advantage of this formalis $m$ that the twistor functions, independent of their analyticity properties, transform as scalars under $\mathrm{SU}(2,2)$. The reason for this, as might be expected, is that the pair $(U, V)$ is actually a generalized "frame" in an appropriate principal bundle, and the main purpose of this paper is to examine this fact in some detail. As a by-product, we are able to give a unified treatment of massless fields, conformally weighted densities, twistor functions, and homogeneous functions on the $\mathrm{O}(2,4)$ null cone, objects which often appear in somewhat different settings.

Some of the representations considered here are quite well known in one form or another, and certain of their properties have been considered at length in the literature. The Lie algebra version of much of this, together with some applications to quantum field theory, may be found in Ferrara et al. ${ }^{3}$ Invariant scalar products are discussed by Gross ${ }^{10}$ and Penrose. ${ }^{5,7}$ These subjects will not be considered here; in particular, we do not discuss the topology of the representations.

The first part of the paper is concerned with establishing the existence of a principal bundle homorphism from $\mathrm{SU}(2,2) \rightarrow N$ onto the set of twistor dyads over $N, N$ being the $O(2,4)$ null cone. It then follows directly that the representations above referred to can all be realized on spaces of twistor functions, different representations being characterized by the homogeneity properties of their respective twistor functions. Those representations which can be analytically continued to the complex $\mathrm{O}(2,4)$ null cone are labeled by an ordered pair of integers $\left(n_{1}, n_{2}\right)$.

We then show that the massless free fields of helicity $n / 2$ belong to an invariant subspace of the $(n, 1)$ representation, and that, in the real analytic case, (1.3) implies (1.1) and (1.2). As a simple application of the formalism introduced, we conclude by showing the direct connection between finite-dimensional representations of $\mathrm{SU}(2,2)$ and solutions to the generalized twistor equation.

\section{PRELIMINARIES}

Notation and conventions:

$\mathrm{M}^{4}$ : Minkowski space; $\eta_{\mu \nu}=\operatorname{diag}\{1,-1,-1,-1\}$

$R: \quad R^{6}$ together with the quadratic form $h_{a b}=\operatorname{diag}\{1$, $-1,-1,-1,-1,1\}$

$N$ : The null cone (minus the vertex) of $R$

$\tilde{M}$ : The space of rays of $N$

$m$ : The space of lines of $N$, the conformal compactification of $\mathrm{M}^{4}$

$T$ : Twistor space; $\mathbf{C}^{4}$ together with the Hermitian form $Q=\operatorname{diag}\{1,1,-1,-1\}$

$\mathbb{C} N$ : The complex $\mathrm{O}(2,4)$ null cone (minus the vertex), defined by the zeroes of $h$ in $\mathrm{C}^{6}$ 
Points of $\mathbf{M}^{4}$ will be denoted either by $x^{\mu}$ or $x^{A B^{\prime}}$, where ${ }^{8}$

$$
\left(x^{A B^{\prime}}\right)=\left(\begin{array}{cc}
x^{00} & x^{01^{\prime}} \\
x^{10^{\prime}} & x^{11^{\prime}}
\end{array}\right) \equiv \frac{1}{\sqrt{2}}\left(\begin{array}{ll}
x^{0}+x^{1} & x^{2}+i x^{3} \\
x^{2}-i x^{3} & x^{0}-x^{1}
\end{array}\right) .
$$

The mapping $\sigma: \mathbf{M}^{4} \rightarrow N$ given by

$$
\sigma\left(x^{\mu}\right)=y^{a}(x) \equiv\left(x^{\mu}, \frac{1}{2}\left[1+x_{\nu} x^{\nu}\right], \frac{1}{2}\left[1-x_{\nu} x^{\nu}\right]\right)
$$

embeds Minkowski space onto the section of $N$ given by the null hyperplane $y^{4}+y^{5}=1$. A useful local coordinate system in $N$ is obtained by letting $x^{\mu}$ label the generator containing $\sigma\left(x^{\mu}\right)$ and defining a fifth coordinate

$$
\kappa=y^{4}+y^{5}, \quad \kappa>0 .
$$

Any slice of $N$ (a four-dimensional submanifold nowhere tangent to the generators) may be given locally by specifying $\kappa$ as a function of the $x^{\mu}$. Using the $x^{\mu}$ as local coordinates in the slice, the induced metric is given by

$$
g_{\mu \nu}(x)=\kappa^{2}(x) \eta_{\mu \nu} .
$$

Thus all conformal rescalings of Minkowski space ${ }^{6,8}$ may be realized as slices of $N$; in particular, taking the slice $\kappa=1$, it follows that $\sigma$ is an isometric embedding, and that $x^{\mu} \in \mathbf{M}^{4}$ can be identified with $\left(x^{\mu}, 1\right) \in N$.

We may also obviously regard $\sigma$ as mapping complex Minkowski space onto the $y^{4}+y^{5}=1$ slice of $\mathbb{C} N$, and a local coordinate system in $\mathbb{C} N$ is obtained by allowing the $x^{\mu}$ to take on arbitrary complex values and $\kappa$ to range over the complex plane minus the negative real axis.

As usual, ${ }^{6,7}$ we choose a basis in $T$ in which $Q$ takes the form

$$
Q=\left[\begin{array}{ll}
0 & 1 \\
I & 0
\end{array}\right] \text {. }
$$

Let $B \in T \wedge T$, and define $y(B) \in \mathbf{C}^{6}$ by

$$
\begin{aligned}
& y^{0}(B)=\frac{-i}{\sqrt{2}}\left(B^{03}-B^{12}\right), \quad y^{3}(B)=\frac{1}{\sqrt{2}}\left(B^{13}+B^{02}\right), \\
& y^{1}(B)=\frac{-i}{\sqrt{2}}\left(B^{03}+B^{12}\right), \quad y^{4}(B)=\frac{1}{2}\left(B^{23}-2 B^{01}\right), \\
& y^{2}(B)=\frac{-i}{\sqrt{2}}\left(B^{13}-B^{02}\right), \quad y^{5}(B)=\frac{1}{2}\left(B^{23}+2 B^{01}\right) .
\end{aligned}
$$

This establishes a one-to-one correspondence between simple twistor bivectors and points of $\mathbb{C} N$. Notice that $y(B)$ is real (lies on $N$ ) only when $B$ is real; i. e., only when $\bar{B}_{\alpha \beta}=B_{\alpha \beta}=\frac{1}{2} \epsilon_{\alpha \beta \gamma \delta} B^{66}$. Two twistors are said to meet at $x=x^{A A^{\prime}}$ if they can be written in the form $U^{\alpha}$ $=\left(i x^{A A^{\prime}} \pi_{A^{\prime}}, \pi_{A^{*}}\right), V^{\alpha}=\left(i x^{A A^{\prime}} \omega_{A^{\prime}}, \omega_{A^{\prime}}\right)$. (see Refs. 11 and 12 for the geometrical implications.) In this case, if $B=2 U \wedge V$,

$$
y(B)=\left(\pi_{A^{*}} \omega^{A^{\prime}}\right) \sigma(x) .
$$

Note, in particular, that $U$ and $V$ may meet at a real point without $2 U \wedge V$ deter mining a point of $N$.

If $y \rightarrow B(y)$ is the inverse of (2.5) and $B(y)$ is regarded as a skew matrix, the action of $\mathrm{SU}(2,2)$ on $N$ is given by

$$
T(y)=y\left(T B(y) T^{t}\right), \quad T \in \mathrm{SU}(2,2) .
$$

The subgroup of $\operatorname{SU}(2,2)$ leaving $\sigma(0)$ fixed is given by

$$
W=\left\{\left[\begin{array}{cc}
\alpha & 0 \\
i A \alpha & \alpha^{*^{-1}}
\end{array}\right]: \alpha \in \mathrm{SL}(2, \mathbb{C}), A \text { Hermitian }\right\} .
$$

Clearly, $W$ is the semidirect product $\operatorname{SL}(2, C) \cdot \mathscr{A}$, where $\mathcal{I}$ is the four-parameter Abelian group of special conformal transformations.

Finally, if $[y]$ denotes the equivalence class of $y \in N$ under the relation $y \sim r y, r \in \mathrm{R}^{+}$, the action of $\mathrm{SU}(2,2)$ on $\widetilde{M}$ is given by

$$
T([y])=[T(y)],
$$

where $y$ is any representative of $[y]$. The subgroup of $\mathrm{SU}(2,2)$ fixing $\sigma(0)$ is $V=\left\{\mathbf{R}^{+} \otimes \mathrm{SL}(2, \mathrm{C})\right\} \circ \boldsymbol{q}$.

\section{REPRESENTATIONS}

We are going to be concerned with representations of $\mathrm{SU}(2,2)$ induced by representations of either $W$ or $V$ with the property that 2 is in the kernel of the inducing representation. Vectors in these representation spaces, regarded as functions on $\mathrm{SU}(2,2)$, will thus be constant on the cosets of $\mathfrak{U}$. It turns out that a particularly useful parametrization of these cosets is provided by pairs of twistors $(U, V)$ satisfying $y(U \wedge V) \in N$.

To see this explicitly, regard a matrix $T \in \mathrm{SU}(2,2)$ as being composed of four twistors

$$
T=\left[\begin{array}{l:l:l:l}
E & F & G & H
\end{array}\right]
$$

subject to $\bar{E}_{\alpha} G^{\alpha}=\bar{F}_{\alpha} H^{\alpha}=1$, all other products vanishing, and $E^{[\alpha} F^{\beta} G^{\gamma} H^{\beta 1}=\epsilon^{\alpha \beta \gamma \delta}$. In the principal bundle $\operatorname{SU}(2,2)$ $\rightarrow \mathrm{SU}(2,2) / W=N, T$ lies in the fiber over

$$
y=y\left(T B(\sigma(0)) T^{t}\right) \text {. }
$$

Since $T B(\sigma(0)) T^{t}=2 G \wedge H$, the third and fourth columns of $T$ determine the fiber in which it lies. Moreover, for

$$
\mathbf{A}=\left[\begin{array}{rr}
I & 0 \\
i A & I
\end{array}\right] E \mathbf{A},
$$

TA has the form $[*: *: G: H]$, so that these same columns are invariant under $\mathcal{X}$ and may be thought of as labeling the elements of $\mathrm{SU}(2,2) / \mathscr{R}$.

We formalize this as follows. Put

$$
S^{*} \equiv\{(U, V) \in T \times T: U \neq \lambda V, \lambda \in \mathbb{C}\} .
$$

Define a projection $P: S^{*} \rightarrow \mathbb{C} N$ by

$$
P(U, V)=y(2 U \wedge V)
$$

and let

$$
S=P^{-1}(N) \text {. }
$$

Notice that for any $y \in \mathbb{C N}, P^{-1}(y) \cong \operatorname{SL}(2, \mathrm{C})$ by virtue of (3.4). It will be convenient to label $U$ and $V$ by spinor indices,

$$
U_{A^{*}}=\left(U_{0^{*}}, U_{1^{*}}\right)=(U, V) .
$$

A right action of $\operatorname{SL}(2, \mathrm{C})$ on $S^{*}$ is given by

$$
U_{A^{\prime}} \cdot \alpha \equiv \bar{\alpha}_{A^{*^{*}}}{ }^{B^{\prime}} U_{B^{\prime}},
$$


where $\bar{\alpha}_{A^{\prime}}{ }^{B^{\prime}}=\overline{\alpha_{A}{ }^{B}}$ and $S^{*} \rightarrow \mathbb{C} N($ resp. $S \rightarrow N$ ) becomes a principal $\mathrm{SL}(2, C)$ bundle. $\mathrm{SU}(2,2)$ acts on $S^{*}$ (resp.

$S)$ in the obvious way,

$$
T \cdot U_{A^{\prime}}=T_{B}^{\alpha} U_{A^{\prime}}^{B} .
$$

Let $\tilde{\rho}:(\alpha, A) \rightarrow \alpha$ be the homorphism from $W$ onto $\operatorname{SL}(2, C)$, and define a mapping $\rho: \operatorname{SU}(2,2) \rightarrow S$ by

$$
\rho:\left[\begin{array}{l:l:l:l}
E & F & G & H
\end{array}\right] \rightarrow(H,-G) .
$$

Evidently, $\rho$ is fiber preserving, and a simple calculation shows that

$$
\rho(R T W)=R \cdot \rho(T) \cdot \tilde{\rho}(W), \quad R, T \in \mathrm{SU}(2,2), \quad W \in W .
$$

Thus, $\rho$ is a principal bundle homorphism which commutes with the action of $\mathrm{SU}(2,2)$.

We come now to the induced representations. Let $\tau: \operatorname{SL}(2, C) \rightarrow \operatorname{aut}(V)$ be a representation of $\mathrm{SL}(2, \mathrm{C})$ on the finite-dimensional vector space $V . \tau$ may be regarded as a representation of $W$ as well, by putting $\tilde{\tau}(\alpha, A)$ $=\tau(\alpha)$. It is an immediate consequence of $(3.10)$ that the two associated homogeneous vector bundles $\mathrm{SU}(2,2)$ $\times_{\tilde{\tau}} V \rightarrow N$ and $S \times_{T} V \rightarrow N$ are isomorphic; thus the representation of $\mathrm{SU}(2,2)$ induced by $\tau$ can be realized on the vector space of cross sections of $S \times{ }_{\tau} V \rightarrow N$. (It is not required that $\tau$ be unitary. )

Recall that an element of $S \times, V$ is an equivalence class $\left\{\left(U_{A^{\prime}}, v\right)\right\}$, with $U_{A^{*}} \in S, v \in V$, where the equivalence relation is defined by

$$
\left(U_{A^{\prime}}, v\right) \sim\left(U_{A^{\prime}}, v\right) \circ \alpha=\left(U_{B^{\prime}}, \bar{\alpha}_{A^{\prime}}^{B^{\prime}}, \tau^{-1}(\alpha) \cdot v\right) .
$$

Let $\Psi$ be a cross section of $S \times_{T} V$, and let $U_{A}, \subseteq S$ with $P\left(U_{A^{\prime}}\right)=y \in N$. Then $\Phi(y)$ has a local representative of the form $\left(U_{A^{\prime}}, \hat{\Phi}\left(U_{A^{\prime}}\right)\right)$, where $\hat{\Phi}\left(U_{A^{\prime}}\right)$ are the "components" of the field $\Phi(y)$ in the twistor dyad $U_{A^{\prime}}$. The function $\hat{\Phi}: S \rightarrow V$ so defined has the property

$$
\hat{\Phi}\left(U_{A^{\prime}} \circ \alpha\right)=\tau^{-1}(\alpha): \hat{\Phi}\left(U_{A^{\prime}}\right),
$$

reflecting the local transformation law of the field, and transforms under $T \in \mathrm{SU}(2,2)$ as

$$
\widehat{T \Phi}\left(T U_{A^{\prime}}\right)=\hat{\Phi}\left(U_{A^{\prime}}\right) \text {. }
$$

(See, for example, Hermann. ${ }^{13}$ ) Conversely, any $V$ valued function on $S$ satisfying (3.12) may be thought of as a cross section of $S x_{T} V$. We ther efore have the result: Any representation of $\mathrm{SU}(2,2)$ induced by $\mathrm{SL}(2, \mathrm{C})$ in the manner described above may be realized on a space of vector-valued functions of two twistor variables $U_{A^{\prime}}$, where $U_{A^{\prime}} \in S$.

\section{HOMOGENEOUS TWISTOR FUNCTIONS}

The representations constructed in the above manner are all highly reducible. The simplest way to see this is to consider $S$ as a bundle over $\widetilde{M}$ with structure group $\mathrm{R}^{+} \otimes \mathrm{SL}(2, \mathrm{C})$ acting in the obvious way,

$$
U_{A^{*}} \cdot r \alpha=r U_{B^{\prime}}, \bar{\alpha}_{A^{\prime}}, \quad r \in \mathrm{R}^{+}, \alpha \in \operatorname{SL}(2, \mathrm{C}) .
$$

Let $\rho^{\prime}: V \rightarrow \mathrm{R}^{+} 8 \mathrm{SL}(2 ; \mathbf{C})$ send $(r \alpha, A)$ to $r \alpha$ and let $\rho$ : $\mathrm{SU}(2,2) \rightarrow S$ be given by (3.9). Then, as before, $\rho$ is a principal bundle homorphism (of bundles over $\bar{M}$ this time) commuting with the action of $\mathrm{SU}(2,2)$. So any representation induced by $\mathrm{R}^{+} \mathrm{SL}(2, \mathrm{C})$ may also be realized on a space of functions of two twistor variables.

In particular, if $\tau$ is any representation of $\mathrm{SL}(2, \mathrm{C})$ on $V$, and $\lambda$ is any complex number, we can define a representation $\tau_{\lambda}: \mathrm{R}^{+} \otimes \mathrm{SL}(2, \mathrm{C}) \rightarrow \operatorname{aut}(V)$ by

$$
\tau_{\lambda}(r \alpha)(v)=\gamma^{\lambda} \tau(\alpha)(v) .
$$

If $\Psi$ is a cross section of $S \times_{\tau_{\lambda}} V \rightarrow \tilde{M}$, the associated twistor function satisfies

$$
\hat{\Psi}\left(U_{A^{\prime}} \cdot r \alpha\right)=r^{-\lambda} \tau^{-1}(\alpha) \cdot \hat{\Psi}\left(U_{A^{\prime}}\right) .
$$

For $r=1$, this agrees with (3.12), and we may therefore regard $\Psi$ (or $\hat{\Psi}$ ) as belonging to an invariant subspace of the representation induced by $\tau$. In short, the mapping $\Phi \rightarrow \hat{\Phi}$ intertwines the $\tau_{\lambda}$ representation with the $\tau$ representation. The net effect of the intertwining operator, as can be seen by taking $\alpha=I$ in (4.3), is to remove the $\mathrm{R}^{+}$dependence (or local scaling behavior) of the original field (defined on $\bar{M}$ ) by spreading it out in an appropriate homogeneous fashion on the generators of N.

Example: Let $V(n)$ be the space of symmetric spinors with $n$ primed indices. Let $\tau(n)$ be the representation of $\mathrm{SL}(2, \mathrm{C})$ on $V(n)$ given by

$$
\tau(n)(\alpha): \xi_{A^{\prime}} \ldots B^{\prime}-\bar{\alpha}_{A^{\prime}}^{-1} C^{\prime} \ldots \bar{\alpha}_{B^{\prime}}^{-1} D^{s} \xi_{C^{\prime} \ldots D^{\prime}}
$$

If $\Phi$ is a cross section of $\int \times_{\tau(n)} V(n) \rightarrow N$, then $\hat{\Phi}$ takes values in $V(n)$ and may be written, somewhat redundantly, as $\hat{\Phi}_{A^{\prime} \ldots B^{*}}\left(U_{C^{\prime}}\right)$. There are $n+1$ independent components here,

$$
\varphi_{r}\left(U_{C^{\prime}}\right)=\underbrace{\hat{\Phi}_{1} \ldots 1}_{r} \underbrace{n_{n} \ldots o r}_{n-r}\left(U_{C^{\prime}}\right), \quad 0 \leqslant r \leqslant n .
$$

If, in addition, $\hat{\Phi}$ satisfies (4.3), the functions $\varphi_{r}$ satisfy

$$
\begin{aligned}
& \varphi_{r}\left(t U_{0}, U_{1}\right)=l^{(n-\lambda-2 r) / 2} \varphi_{r}\left(U_{o}, U_{1}\right), \\
& \varphi_{r}\left(U_{0}, l U_{1}\right)=l^{(-n-\lambda+2 r) / 2} \varphi_{r}\left(U_{0}, U_{1}\right)
\end{aligned}
$$

for $l>0$. [To obtain (4.6a), for example, note that $\left(t U_{n}\right.$, $\left.U_{1},\right)=\left(U_{n}, U_{1}\right) \cdot\left(t^{1 / 2} \alpha\right)$, with $\alpha=\left[\begin{array}{cc}t^{1 / 2} & 11 \\ 0 & t=1 / 2\end{array}\right]$, and use $(4.3)$ and $(4.5) .1$

Though no assumption has yet been made concerning the smoothness of $\Phi$, it is natural within the twistor formalism to consider the possibility that the functions given in (4.6) are actually the restrictions to $S$ of singlevalued holomorphic functions defined on open sets of $S^{*}$. A necessary precondition for this is that the exponents appearing in (4.6) be integers; if $\lambda$ is set equal to $n+2 k$, this means that $k$ must be an integer. In this case, independent of analyticity properties, $\hat{\Phi}$ is called a function of type $(n, k)$. It will be shown in the next section that a massless free field of helicity $n / 2$ is a function of type $(n, 1)$.

Clearly, both $S^{*}$ and $S^{*} \times_{\tau(n)} V(n)$ are holomorphic bundles over $\mathbb{C} N$, and a holomorphic (local) cross section of $S^{*} \times_{T(n)} V(n)$ determines a holomorphic $V(n)$ valued function of $U_{A}$, satisfying (3.12). In this context, if a $V(n)$-valued function $\$$ [of type $(n, k)$, say] is the restriction to $W \subseteq S$ of a function holomorphic in some open neighborhood of $W$ in $S^{*}$, we call the field $\Phi$ determined by $\hat{\Phi}$ real analytic on $P(W) \subseteq N$. It will be evident in the next section that this corresponds to the usual 
notion of real analyticity whenever it makes sense. Notice that fields of type $(n, k)$ which are real analytic on the whole of $N$ are mapped onto themselves by the action of $\mathrm{SU}(2,2)$. Examples of such fields can be obtained by contour integration from Penrose's "elementary states." 6

To continue, we now wish to show that if $\hat{\Psi}$ satisfies (4.3), the components of $\Psi$ (regarded as a cross-section of $S x_{T} V \rightarrow N$, will actually be homogeneous functions of $r$ in suitably chosen bases. Let $y \rightarrow G(y)$ be a local cross section of $\mathrm{SU}(2,2)$ over $N . G$ is called elementary if, whenever $y$ and $r y(r>0)$ are in the domain of $G, G(r y)$ $=G(y) D(r)$, with

$$
D(r)=\left[\begin{array}{cc}
r^{-1 / 2} I & 0 \\
0 & r^{1 / 2} I
\end{array}\right] .
$$

Correspondingly, $\rho(G(y))$ is called an elementary cross section of $S$. Denoting $\rho(G(y))$ by $U_{A^{e}}(y)$, we have

$$
U_{A^{*}}(r y)=r^{1 / 2} U_{A^{*}}(y) \text {. }
$$

Thus, except for a scale factor, such cross sections are constant on the generators of $N$.

As was mentioned before, a cross section of $S \times_{\tau} V$ $\rightarrow \widetilde{M}$ may be regarded as a cross section of $S \times, V \rightarrow N$ as well. The components of $\Psi(y)$ in the dyad $U_{A},(y)$ are given by

$$
\psi(y)=\hat{\Psi}\left(U_{A^{*}}(y)\right),
$$

and by construction, it is immediate that (compare Refs. $1-3$ )

$$
\psi(r y)=r^{-\lambda / 2} \psi(y) .
$$

From a slightly different point of view, the same cross section may be realized as a conformally weighted density in $\mathbf{M}^{4}$ (compare Refs. 8, 14, and 15). When expressed in terms of $x^{\mu}$ and $\kappa$, the components of $\psi$ are are homogeneous of degree 0 in the $x^{\mu}$ and of degree $-\lambda / 2$ in $\kappa$. Thus, if $M$ is a slice of $N$ given by $\kappa(x)$, the components of $\psi$ on $M$ are related to those on $\mathbf{M}^{4}$ by

$$
\psi\left(x^{\mu}, \kappa(x)\right)=[\kappa(x)]^{-\lambda / 2} \psi\left(x^{\mu}, 1\right) .
$$

Identifying $M$ with $\mathbf{M}^{4}$ via $(x, \kappa(x)) \leftrightarrow(x, 1)$, this can be written as

$$
\psi^{\prime}(x)=[\kappa(x)]^{-\lambda / 2} \psi(x) .
$$

Together with (2.3), this gives the local transformation law for a density of conformal weight $-\lambda / 2$. Of course, if the cross section of $\mathrm{SU}(2,2)$ is not elementary, the right-hand side of (4.12) will contain an $x$-dependent SL $(2, \mathrm{C})$ matrix acting on the indices of $\psi$.

\section{THE ZERO REST-MASS FIELDS}

Consider a solution to the helicity $n / 2$ free field equations in $\mathbf{M}^{4}$,

$$
\nabla^{A A^{\prime}} \varphi_{A^{\prime} B^{\prime} \ldots C^{\prime}}=0
$$

with $\varphi_{A^{\prime} \ldots C^{\prime}}$, being the components, in the standard frame, of a spinor field with $n$ indices. We can associate with $\varphi$ a twistor function of type $(n, 1)$ by declaring that $\varphi_{A^{\prime} \ldots C^{\prime}}$ are to be the components at $(x, 1) \in N$ of $\hat{\Phi}$ in a particular basis.
Define the standard cross section $\hat{U}_{A^{\prime}}(x, \kappa)$ of $S \rightarrow N$ to be the image, under $\rho$, of

$$
G(x, \kappa)=T(x) D(\kappa)=\left[\begin{array}{cc}
I & i x^{A A^{*}} \\
0 & I
\end{array}\right]\left[\begin{array}{cc}
\kappa^{-1 / 2} I & 0 \\
0 & \kappa^{1 / 2} I
\end{array}\right] .
$$

(In $\mathbf{M}^{4}$, this is the normal basis in which the operators $P^{\mu}$ do not act on the indices of the fields.) We thus have

$$
\begin{aligned}
\hat{U}_{J^{*}}\left(x^{\mu}, \kappa\right) & =(\kappa)^{1 / 2}\left(i x^{C D^{\circ}} o_{D^{\prime}}, o_{D^{*}} ; i x^{C D^{\prime}} i_{D^{*}}, \imath_{D^{\prime}}\right) \\
& =(\kappa)^{1 / 2} \hat{U}_{J^{*}}\left(x^{\mu}, 1\right),
\end{aligned}
$$

where $o_{D^{\prime}}=(0,1)$ and $i_{D^{\prime}}=(-1,0)$ are the components of the standard spin-frame at $x$. Allowing $x$ to take on arbitrary complex values and $k$ to range over the complex plane minus the negative real axis, we may extend this to a holomorphic local cross section of $S^{*}-\mathbb{C N}$ (sitting over an open neighborhood of $\mathbf{M}^{4} \times \mathrm{R}^{+}$in $\mathbb{C} N$ ) by demanding that $(5.3)$ continue to hold.

Restricting our attention to $S \rightarrow N$ for the moment, we now define $\hat{\Phi}$ by

$$
\hat{\Phi}_{A^{\alpha}{ }^{\alpha} \ldots B^{\prime}}\left(U_{K^{\prime}}(x, \kappa)\right)=\kappa^{-n / 2-1} \varphi_{A^{\prime} \ldots B^{*}}(x),
$$

the value of $\hat{\Phi}$ in any other twistor dyad over $(x, k)$ being given by (3.12). Notice that if

$$
U_{K^{\prime}}=\bar{\alpha}_{K^{\prime}}{ }^{\prime \prime} \hat{U}_{J^{\prime}}(x, 1)
$$

is an arbitrary twistor dyad at $x$, it has the form

$$
U_{\sigma^{\prime}}=\left(i x^{C D^{\prime}} \tilde{o}_{D^{\prime}}, \tilde{o}_{D^{\prime}}\right), \quad U_{1^{\prime}}=\left(i x^{C D^{\prime}} \tilde{\imath}_{D^{\prime}}, \tilde{\imath}_{D^{\prime}}\right) \text {, }
$$

where

$$
\tilde{o}_{D^{\prime}}=\left(\bar{\alpha}^{-1}\right)_{D^{\prime}}{ }^{C^{\prime}} o_{C^{\prime}}, \tilde{z}_{D^{\prime}}=\left(\bar{\alpha}^{-1}\right)_{D^{\prime}}{ }^{C^{\prime}} l_{D^{\prime}} .
$$

Using (3.12), one sees that $\hat{\Phi}\left(U_{K^{\prime}}\right)$ are the components at $x$ of the original field in the spin-frame $\{\tilde{o}, \tilde{\imath}\}$. Thus the dyad $U_{K}$, determines both the point $x$ and a spin-frame at $x,{ }^{5}$ while the transformation properties of $\hat{\Phi}$ correctly mirror the behavior of $\varphi$ under local SL $(2, C)$ transfor mations. ${ }^{16}$

To transform the original field $\varphi$ under $\operatorname{SU}(2,2)$ is now a simple matter. If $T \in \mathrm{SU}(2,2)$, we define

$$
(T \varphi)_{A^{\prime} \ldots B^{\prime}}(x)=\widehat{T \Phi}_{A^{\prime} \ldots B^{\prime}}\left(\hat{U}_{K^{\prime}}(x, 1)\right) .
$$

It is now easy to verify that

$$
\nabla^{A A^{\prime}}(T \varphi)_{A^{\prime} B^{\prime} \ldots C^{\prime}}=0,
$$

i.e., that Eq. (5.1) is conformally invariant. Poincare and dilation invariance is immediate, so the only thing that needs to be checked is invariance under $\mathfrak{A}$. Since $\mathscr{U}$ is conjugate to the translations under $H=\left[\begin{array}{ll}0 & I \\ I & 0\end{array}\right]$, the proof reduces to showing that $H \varphi$ satisfies (5.1) if $\varphi$ does. The calculation involved is straightforward, but quite lengthy, and is omitted, although it should be noted that this is the point at which the precise degree of homogeneity of $\hat{\Phi}$ plays a crucial role.

Conversely, it is clear that any $\hat{\Phi}$ of type $(n, 1)$ which satisfies (5.1) in the standard cross section gives rise to a zero rest-mass field in $\mathbf{M}^{4}$. In a general cross $\mathbf{s e c -}$ tion (restricted to $\mathrm{M}^{4}$ ), Eq. (5.1) will of course involve the spinor covariant derivative.

We now confine our attention to solutions of $(5,1)$ which are real analytic on some open subset of $\mathrm{M}^{4}$. These admit holomorphic extensions to open subsets of 
$\mathbf{C M}^{4}$ which also satisfy (5.1). Using (5.4) with $x$ and $\kappa$ complex, we obtain a $\hat{\Phi}$ of type $(n, 1)$, holomorphic on some domain $D \subseteq S^{*}$, where $D \cap S$ is open in $S$. Thus, from (5.4),

$$
\nabla^{A A^{\prime}} \hat{\Phi}_{A^{\prime} B^{\prime} \ldots C^{\prime}}\left(\hat{U}_{J^{\circ}}(x, \kappa)\right)=0 .
$$

The two equations $\left(A^{\prime}=0^{\prime}, 1^{\prime}\right)$ reduce to

$$
\frac{\partial \varphi_{r}}{\partial U^{0}}+\frac{\partial \varphi_{r+1}}{\partial V^{0}}=\frac{\partial \varphi_{r}}{\partial U^{1}}+\frac{\partial \varphi_{r_{+1}}}{\partial V^{1}}=0
$$

Although these equations apparently hold only on the standard cross section of $S^{*}$, they are in fact valid on the entire domian of $\hat{\Phi}$. To see this, let $\alpha \in \mathrm{SL}(2, C)$ and consider the cross section $\tilde{U}_{J},(x, k)=\bar{\alpha}_{J^{\prime}}{ }^{\prime} \hat{U}_{K^{\prime}}(x, \kappa)$. Then (3.12) together with the local SL $(2, \mathrm{C})$ invariance of the fields, implies

$$
\widetilde{\nabla}^{A A} \hat{\Phi}_{A^{\prime} B^{\prime} \ldots C^{\prime}}\left(\tilde{U}_{J^{\prime}}(x, \kappa)\right)=0,
$$

where $\tilde{\nabla}^{A A^{\prime}}=\bar{\alpha}_{C^{\prime}}^{-1} A^{\prime} \nabla^{A C^{\prime}}$. Expanding (5.12) then gives (5.11) for $U_{J}=\tilde{U}_{J}$. Since any dyad over $(x, \kappa)$ may be obtanied in this fashion, $(5.11)$ is valid on an open subset of $S^{*}$; thus, by analytic continuation, $(5.11)$ holds whenever it makes sense.

Now let $H$ act on $\hat{\Phi}$ : this interchanges $U^{0}$ with $U^{2}$ and $U^{1}$ with $U^{3}$ (similarly for $V^{\alpha}$ ). Since $H \varphi$ satisfies (5.10), proceeding as above we obtain the additional equations

$$
\frac{\partial \varphi_{r}}{\partial U^{2}}+\frac{\partial \varphi_{r+1}}{\partial V^{2}}=\frac{\partial \varphi_{r}}{\partial U^{3}}+\frac{\varphi_{r+1}}{\partial V^{3}}=0 .
$$

We therefore have

$$
\frac{\partial \varphi_{r}}{\partial U^{\alpha}}+\frac{\partial \varphi_{r+1}}{\partial V^{\alpha}}=0, \quad r=0,1, \ldots, n-1 .
$$

The homogeneity conditions (4.6) may be written

$$
U^{\alpha} \frac{\partial \varphi_{r}}{\partial U^{\alpha}}=-(r+1) \varphi_{r}, \quad V^{\alpha} \frac{\partial \varphi_{r}}{\partial V^{\alpha}}=-(n-r+1) \varphi_{r},
$$

and these are the twistor versions of the zero rest-mass free field equations. ${ }^{5-7}$ The auxilliary conditions

$$
\frac{\partial^{2} \varphi_{r}}{\partial U^{[\alpha} \partial V^{\beta 1}}=0
$$

follows immediately from (5.14).

Conversely, Eqs. (5.14)-(5.16) are manifestly conformally invariant, and it is a simple matter to check that any solution to them yields, in the manner described above, a real analytic solution to the zero rest-mass field equations in Minkowski space. If the functions $\varphi_{r}$ are holomorphic on a neighborhood of the whole of $S$ in $S^{*}$, the resultant field will be single-valued and vanish in a characteristic fashion at null infinity. Specifically, it will exhibit the "peeling-off" property; see Ref. 8 and the other references cited there.

Remark: The center of $\mathrm{SU}(2,2)$ is generated by powers of $J=\left[\begin{array}{cc}i r & 0 \\ 0 & i r\end{array}\right]$. For twistor functions of type $(n, 1)$,

$$
J \varphi_{r}=(-i)^{-n-2} \varphi_{r}
$$

Thus, if $n \equiv 2(\bmod 4), J \varphi_{r}=\varphi_{r}$, and we have a representation of the confor mal group. If $n \equiv 0(\bmod 4)$, we have a representation of $\mathrm{SO}_{0}(2,4)$, while if $n \equiv 1$ or $3(\bmod 4)$, we have a representation of $\mathrm{SU}(2,2)$.

\section{COMPLEX CONJUGATE REPRESENTATIONS}

The complex conjugates of the representations considered above are most usefully realized on the bundle of dual twistor dyads. Let

$$
S^{* *}=\left\{\left(U_{\alpha}, V_{\alpha}\right): U_{\alpha} \neq \lambda V_{\alpha}, \lambda \in \mathbf{C}\right\}
$$

with $U$ and $V$ transforming under the adjoint representation of $\mathrm{SU}(2,2)$. Letting $\bar{U}^{\alpha}=Q^{\alpha \beta} U_{\beta}$, define the projection from $S^{* *}$ to $\mathbb{C N}$ by

$$
P^{\prime}\left(U_{\alpha}, V_{\alpha}\right)=P\left(\bar{U}^{\alpha}, \bar{V}^{\alpha}\right) \text {. }
$$

Denote the pair $\left(U_{\alpha}, V_{\alpha}\right)$ by $U_{A}(A=0,1)$, and define the action of $\mathrm{SL}(2, C)$ by

$$
U_{A} \cdot \alpha=\alpha_{A}{ }^{B} U_{B} \cdot
$$

The bundle of dual twistor dyads over $N$ is defined by $S^{\prime}=P^{\prime-1}(N)$.

Just as above, the representation of $\mathrm{SU}(2,2)$ induced by the representation

$$
\pi(n)(\alpha): \xi_{A \cdots B} \rightarrow \alpha_{A}^{-1} C \cdots \alpha_{B}^{-1 D} \xi_{C \cdots D}
$$

of $\mathrm{SL}(2, \mathrm{C})$ may be realized as a space of functions of the two variables $U_{A}$. It is a simple matter to verify that the homogeneous vector bundle constructed in this fashion is (isomorphic to) the complex conjugate of $S \times_{T(n)} V(n)$. There is an analogous decomposition of the representation into homogeneous twistor functions. In particular, corresponding to a real analytic solution of the field equations

$$
\nabla^{A A^{\prime}} \psi_{A B \cdot . \circ C}=0 \text {, }
$$

there is a holomorphic twistor version, ${ }^{6,7}$

$$
\begin{aligned}
& \frac{\partial \psi_{r}}{\partial U_{\alpha}}+\frac{\partial \psi_{r+1}}{\partial V_{\alpha}}=0 \\
& U_{\alpha} \frac{\partial \psi_{r}}{\partial U_{\alpha}}=-(r+1) \psi_{r}, \quad V_{\alpha} \frac{\partial \psi_{r}}{\partial V_{\alpha}}=-(n-r+1) \psi_{r} .
\end{aligned}
$$

The field $\bar{\psi}_{A^{\prime} \ldots B^{\prime}}=\bar{\psi}_{A \cdots B}$ determines (see Sec. 5) a set of twistor fucntions $\left\{\varphi_{r}\left(U^{\alpha}, V^{\alpha}\right)\right\}$ satisfying $(5.14)-(5.16)$. They are related to the $\psi_{r}$ 's by

$$
\varphi_{r}\left(\overline{U^{\alpha}}, \bar{V}^{\alpha}\right)=\overline{\psi_{r}\left(U_{\alpha}, V_{\alpha}\right)} \text {. }
$$

\section{FINITE-DIMENSIONAL REPRESENTATIONS}

In certain applications, ${ }^{6,7,11}$ it is convenient to regard a twistor $W_{\alpha}=\left(w_{A}, \pi^{A^{\prime}}\right)$ of valence $\left[\begin{array}{l}0 \\ 1\end{array}\right]$ as defining a spinor field in Minkowski space via

$$
\sigma_{A^{*}}(x) \equiv i x_{A A^{*}} \cdot w^{A}-\pi_{A^{*}}
$$

This field satisfies the twistor equation ${ }^{11}$

$$
\nabla_{B\left(C^{\prime}\right.}, \sigma_{\left.A^{\prime}\right)}=0
$$

Conversely, it can be shown that any global solution to (7.2) is of the form (7.1), thereby uniquely defining a twistor $W_{\alpha}$.

Equations such as (7.1) arise quite naturally in the formalism given in this paper. For consider the constant, totally symmetric twistor $S_{\alpha \beta \ldots \gamma}$ of valence $\left[\begin{array}{l}0 \\ n\end{array}\right]$. This can be used to define a symmetric spinor-valued function $\Sigma_{A^{*} \ldots B^{*}}$ on $S^{*}$, 


$$
\Sigma_{A^{\prime} \cdots B^{\prime}}=S_{\alpha_{0,0}} U_{A^{\prime}}^{\alpha} \cdots U_{B^{\prime}}^{\beta} \text {. }
$$

Upon writing out the independent components of $\Sigma$, one sees that it is a function of type $(n,-n)$; thus this representation contains a finite-dimensional invariant subspace. If we use $\Sigma$ to define a conformally invariant field in $\mathbf{M}^{4}$ by putting

$$
\tau_{A^{\prime} B^{\prime} \ldots K^{\prime}}(x) \equiv \Sigma_{A^{\prime} B^{\prime} \ldots K^{\prime}}\left(\hat{U}_{J^{\prime}}(x, 1)\right),
$$

then an easy calculation gives

$$
\nabla_{E\left(E^{\prime}\right.} \tau_{\left.A^{\prime} B^{\prime} \ldots K^{\prime}\right)}=0 \text {, }
$$

a solution to the generalized twistor equation. Conversely, using the methods of Sec. 5, it may be shown that any solution to (7.5) uniquely determines a symmetric twistor of valence $\left[\begin{array}{l}0 \\ n\end{array}\right]$.

\section{CONCLUSION}

We have established a correspondence between a certain series of induced representations of $\mathrm{SU}(2,2)$ and different space of homogeneous functions of two twistor variables. We have shown, in a concrete way, that the zero rest-mass fields, homogeneous functions on $N$, and conformally weighted spinor densities, all of which naturally occur in discussions of the conformal group, should be regarded as representing different aspects of the same geometric objects, namely cross sections of homogeneous vector bundles. We have also tried to show that, even in the nonanalytic case, these objects are most naturally described by means of twistors. The advantages of the twistor description are particularly evident in the case of analytic fields.

As a final remark, we should note that while twistor theory is manifestly conformally invariant, it is a simple matter to "break conformal invariance" and thereby obtain a Poincare invariant theory. It is only necessary to restrict the bundles $S$ and $S^{\prime}$ to the slice $k=1$ of $N$ and it is achieved in practice by incorporating the "infinity twistor" into the equations. For this, and other matters related to quantization, the reader is referred particularly to Ref. 7 .

* Partially supported by the General Research Fund of the University of Kansas.

${ }^{1}$ P. A. M. Dirac, Ann. Math. 37, 429 (1936).

${ }^{2} \mathrm{G}$. Mack and A. Salam, Ann. Phys. 53, 174 (1969).

${ }^{3}$ S. Ferrara, R. Gatto, and A. F. Grillo, Springer Tracts in Modern Physics, Vol. 67 (1973).

${ }^{4}$ T.H. Go and D.H. Mayer, Rep. Math. Phys. 5, 187 (1974).

${ }^{5} \mathrm{R}$. Penrose, Int. J. Theor. Phys. 1, 61 (1968).

${ }^{6}$ R. Penrose and M. A.H. MacCallum, Phys. Rep. 6C, 242 (1973).

${ }^{7} \mathrm{R}$. Penrose in Quantum Gravity: An Oxford Symposium (Oxford U. P., 1975).

${ }^{8}$ R. Penrose, Proc. Roy. Soc. London A 284, 159 (1965)

${ }^{9}$ B.D. Bramson, R. Penrose, and G.A.J. Sparling, "A Derivation of the Twistor Description of Free Fields," to appear.

${ }^{10}$ L. Gross, J. Math. Phys, 5, 687 (1964).

11R. Penrose, J. Math. Phys. 8, 345 (1967).

${ }^{12}$ R. Hansen and E. T. Newman, Gen. Rel. Grav. 6, 361 (1975).

${ }^{13} \mathrm{R}$. Hermann, Lie Groups for Physicists (Benjamin, New York, 1966).

${ }^{14}$ H. A. Buchdahl, Nuovo Cimento 11, 496 (1959).

${ }^{15} \mathrm{R}$. Penrose in Group Theory in Nonlinear Problems, edited by A. Barut (Reidel, Holland, 1974).

${ }^{16}$ The identification of a twistor dyad with an $\mathrm{SL}(2, \mathrm{C})$ spinframe can only be made over simply connected regions of $N$. Any attempt to extend this identification further leads to inconsistencies (the "Grgin discontinuities"7).

${ }^{17} \mathrm{E}$. Grgin, Ph. D. Thesis, Syracuse University (1966).

${ }^{18} \mathrm{D}$. Lerner, "Global Properties of Conformally Invariant Fields," to appear. 